ISSN 1641-6414

DIAGNOSTYKA, 2021, Vol. 22, No. 2

e-ISSN 2449-5220

DOI: $10.29354 /$ diag/137915

\title{
NONDESTRUCTIVE DIAGNOSTICS AND DETECTION OF SUBSURFACE CRACKS IN NON-RIGID PAVEMENTS WITH GPR
}

\author{
Dmitry O. BATRAKOV ${ }^{1}$, Angelika G. BATRAKOVA ${ }^{2}$, Sergey N. URDZIK ${ }^{2}$, Vadim R. DANIELYAN ${ }^{2}$ \\ ${ }^{1}$ Department of Theoretical Radiophysics V. N. Karazin Kharkiv National UniversityKharkiv, Ukraine \\ batrakov@karazin.ua, \\ ${ }^{2}$ Chair of Highway Design, Geodesy and Land Management, Kharkiv National Automobile and Highway \\ University, Kharkiv, Ukraine agbatr@ukr.net
}

\begin{abstract}
The results of laboratory experiments that show the capabilities of modern georadars are presented. Analysis of GPR capabilities allowed to propose a new approach to solving the actual problem of detecting subsurface cracks. The proposed method for recording pulse signals and data processing is based on algorithms and software previously developed by the authors. With the help of this method and the experiments carried out, the possibility of agreed solution to the problem of choosing the optimal parameters of the GPR pulsed signals was shown. To confirm the effectiveness of the developed signal processing methods, a series of laboratory experiments were carried out. The new version of the program "GeoVizy2020" made it possible to increase the efficiency of processing complex impulse signals, as well as to realize the possibility of calculating the quantitative values of the most important parameters of the road pavement layers - the relative permittivity and thickness. Also, to check the adequacy of the results obtained, a method was used, which is based on the procedure for direct measurement of the real layer thickness in laboratory and field conditions and comparison of the data obtained with the results of numerical simulation. As a result, evidence is presented of the advantages of using pulsed signals for assessing layer parameters, searching for subsurface cracks and processing the results of surveys of road surfaces is presented.
\end{abstract}

Keywords: georadar, non-rigid pavement, roads assessment, pulse signals, subsurface cracks

\section{INTRODUCTION}

The problem of assessing the current condition of highways is relevant due to the high level of costs for their repair and maintenance [1-3]. This problem stimulates the solution of the problems of developing methods for assessing various mechanical and geometric parameters, as well as diagnosing various distress in the road pavements, since this is the most material-intensive and expensive part of the road structure. The geometric parameters of road pavements usually refer to the values of the thickness of the layers $[4,5]$, the mechanical properties address their ability to withstand repeated loads [6]. The most common defects in road pavements include cracks, delamination, potholes, rutting, and others [7]. Cracks, including subsurface cracks, are considered the most dangerous defects, since if they are untimely serviced, more serious problems appear, up to the destruction of the pavement [8, 9]. Therefore, the main goal of this work can be formulated as follows: analysis of the existing means of obtaining information about cracks in the road surface and methods of processing the initial data and on this basis improving on this basis the approach to solving the problems of non-destructive testing. The main object of the given research is non-rigid road pavements. To achieve this goal, it is necessary to solve several related tasks. The first task is to select the optimal technical means for obtaining the initial information and technical characteristics of control devices. The second task is related to the development of effective methods for processing the received data. Finally, the third task is to establish the relationships of the obtained data with the technical characteristics of road pavements. Under the term technical characteristics of road pavements, we mean parameters that are of direct interest to the main consumer of this scientific product - the road engineer. The road engineer is primarily interested in assessing the ability of the road pavement to resist traffic loads and climatic factors. Correct assessment allows predicting the service life of the pavement before repair and makes it possible to estimate the cost of maintenance, as well as of ongoing and major repairs. Advances in computer technology and progress in the field of non-destructive testing tools form the basis for setting and solving these problems. We emphasize that a universal means of solving problems of remote sensing and nondestructive testing has not yet been proposed. Therefore, based on the logic of the research, the work has the following structure. The first section contains an analysis of literary sources and a 
problem statement. The purpose of this analysis is to implement the formulation of the problem, taking into account the contradictory requirements for the signal parameters, and, consequently, for the antenna characteristics. In the next section, a new algorithm for processing remote sensing data is proposed, based on the results of earlier works by the authors. The results of laboratory and field studies carried out by the authors are also presented. In the conclusion, the possible ways of development of this scientific direction are formulated for solving urgent problems of the road industry - ensuring the normative technical and operational conditions of road pavements.

\section{SHORT LITERATURE REVIEW AND PROBLEM STATEMENT}

\subsection{Literature review}

An effective solution to the tasks set can be proposed only on the basis of an analysis of the capabilities of existing technical means and methods of processing the received data. The existing limitations, which are caused by the physical capabilities of the methods or by the technical parameters of the means of control, encourage the search for new approaches. For example, the use of such reliable means of control as coring or cutting in certain areas is associated not only with high costs, but also with the inability to carry out continuous monitoring along the road surface $[10,11]$. Today reliable control of pavements is mainly based on examining core specimens, i.e. destructive test method that relies on sparse evaluated values [15]. A similar situation exists in the case of using shock waves to control the condition of the pavement, and applying appropriate processing methods. The most common tool here is the Falling Weight Deflectometer (FWD) [12, 13]. Methods based on the excitations of acoustic waves in the investigated medium with subsequent processing of the wave image also received widespread use. $[14,15]$. The advantage of these methods is the presence of a direct relationship between the mechanical properties of the material: Young's modulus E, Lamé's constants, the material density and its Poisson's ratio on the one hand, and different waves parameters on the other.

Wave parameters primarily include their propagation velocity, complex dispersion curves, group velocity and spectral parameters [15]. The most significant disadvantage of this approach is the need for a primary point action to excite wave processes. This leads to the impossibility of performing the control procedure while the laboratory is moving.

At the same time, it was noted in $[16,17]$ that ground penetrating radars (GPR) allow obtaining primary data when a laboratory car moves at the speed of a traffic flow. As noted in [18-22], the use of GPR allows solving both the problem of assessing the geometric and electrical parameters of road pavements, and the problem of detecting defects in them. It should be noted that the assessment of the geometric parameters of the layers is, on the one hand, an independent stage in monitoring the road pavement condition, and on the other hand, it serves as the basis for the search for defects. Earlier, we proposed an approach to the problem of detecting and evaluating the parameters of cracks - opening width, depth and filling [9]. This approach was based on obtaining initial data using two types of antenna units. The first antenna unit was a system of transmitting and two receiving antennas, described in detail in [23]. The second block included two Vivaldi planar slot antennas and was intended for registration of cross-polarization components. As the experience of practical use of the first unit has shown, the antenna unit with two receiving antennas has a disadvantage, which is associated with the presence of two directions of radiation and receiving signals. It follows that a prerequisite for its use is the absence of foreign objects (alien artifacts) in the space above the antennas. It is not always possible to ensure the fulfillment of this condition. Therefore, a new antenna unit with dipole antennas of two configurations, bistatic and cross-polarized positions, was created, and a data processing algorithm was proposed [24]. In addition, there is a patent of Ukraine [25], which involves the excitation of two alternately arranged mutually orthogonally linearly polarized emitters of the transmitting antenna with switchable polarization. They emit an electromagnetic field, first with one polarization, and then with orthogonal linear polarization, and receive the reflected signal with two linearly polarized receiving antennas. The polarization directions of these antennas are oriented at an angle of 60 degrees to each other. After detecting the received signals, they are amplified by narrow-band AC (alternative current) voltage amplifiers, which are tuned in advance to the switching frequency of the emitters. The amplified signals are converted into corresponding digital codes with the help of multi-bit analog-todigital converters and, with the help of computer programs, the dependence of the amplitudes of the received signals on the polarization directions of the emitters and receiving antennas is analyzed. The conclusion on the presence of cracks in the asphalt concrete pavement is made in case there is a difference between the amplitudes of the signals received simultaneously by two receiving antennas. The direction of the crack is determined by the difference in the amplitudes of these signals. However, as follows from the above description, this diagnostic method is associated with the need to use several antennas at the same time. They must also be oriented in space in a fixed way - at strictly specified angles. Obviously, these requirements can make this approach difficult to apply. Therefore, in this work, we will focus on the analysis of the 
possibilities of practical use of the simplest bistatic configuration of the antenna unit from a technical point of view. The physical basis for this approach is also the well-known fact of visual display of subsurface cracks and other linear inhomogeneities in the form of hyperbolas [26, 27].

\subsection{Problem statement}

To achieve presentation clarity, let us consider in more detail problem statement. Consider a pavement model in its most general form - a flatlayered medium, which consists of several homogeneous layers. Without loss of generality, we will assume that the medium consists of 3 layers located on a semi-infinite substrate. This is because the standard pavement structure consists of two layers of asphalt, a layer of reinforced materials, a base and a subgrade. Cracks, including subsurface ones, can only be in the layers of asphalt concrete. The lower layers of the so-called disconnected materials have relatively close values of the dielectric constant. Therefore, we assume that the crack is located in the lower layer of asphalt concrete and has a rectangular cross section (Fig. 1). As the main initial data for solving the problem of detecting and positioning (determining the direction) of a crack, we will take the results of a GPR survey. In other words, we will assume that in the process of obtaining the primary data sets, a preliminary survey of this road section was carried out using a pulse GPR, for example the Odyag GPR $[9,23]$. Antenna unit of impulse georadars, as a rule, contains two antennas - transmitting (A1 - in Fig. 1) and receiving (A2 - in Fig. 1). These antennas can generally be oriented at an angle to each other. The angle usually has a value in the range from zero to 30 . Thus, the task of detecting cracks, including subsurface ones, within the framework of this approach is reduced to developing an algorithm for analyzing the received pulse signals, or otherwise, the so-called carrierless signals. This term implies that the antenna records directly the change in the parameters of the electromagnetic field, and not the change in the amplitude of the carrier frequency, as in in the case of classical radar.

Before proceeding to the description of the proposed approach, we we'd like to note that the standard method for processing sound data is a visual assessment of radarograms by an operator. The term radarogram refers to an image of a set of temporal signals obtained using a variable density display. In this case, the path length is plotted along the horizontal axis, time along the vertical axis, and the signal amplitude is displayed at each point. The display can be either color or black and white. As an example, there is a radarogram obtained on one of the roads in Ukraine. The peculiarity of this radarogram is that it was obtained while driving over a narrow transverse metal structure at the base of the bridge. The radarogram clearly shows hyperboles at the beginning of the passage over the bridge and at the end of the passage Fig. 2 . Approximate methods can be used to analyze the data obtained [28]. One of the possible variants of this approach is based on the assumption that the crack is located in the lower layer of asphalt concrete (the second layer of coverage) and has relatively small ratio of thickness to height - no more than 0.5. The thickness of the upper layer of the pavement, which at this stage is assumed to be unknown, is denoted by $h_{1}$, and the dielectric constant of the upper layer is $\varepsilon_{1}$. For the second pavement layer, respectively $-h_{2}, \varepsilon_{2}$.

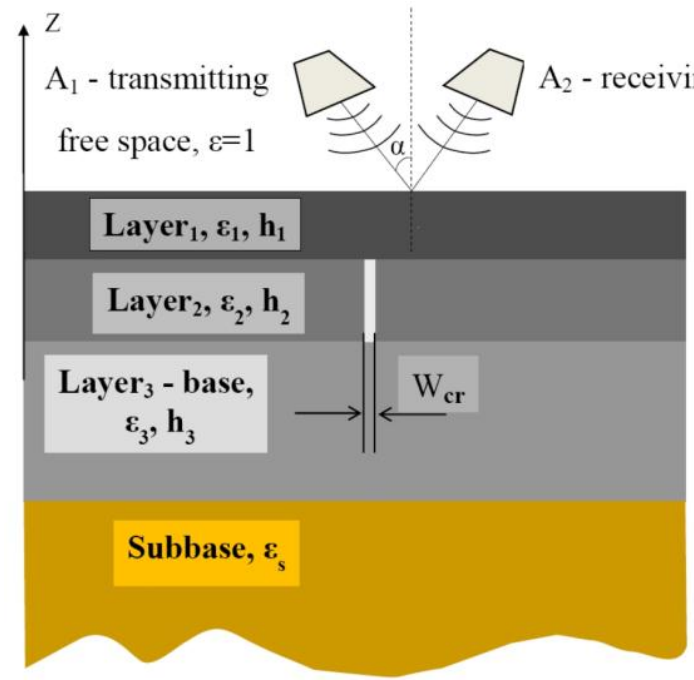

Fig. 1. Problem geometry

We denote $d$ the height of the antenna unit location above the upper boundary of the coverage as $d$ and assume that two measurements have been made - one when the antenna is located strictly above the crack $\left(\mathrm{O}_{1}\right.$ Fig. 3), and the second when the antenna is displaced $\Delta R$ (horizontally) from the first position $\left(\mathrm{O}_{2}\right.$ Fig. 3).

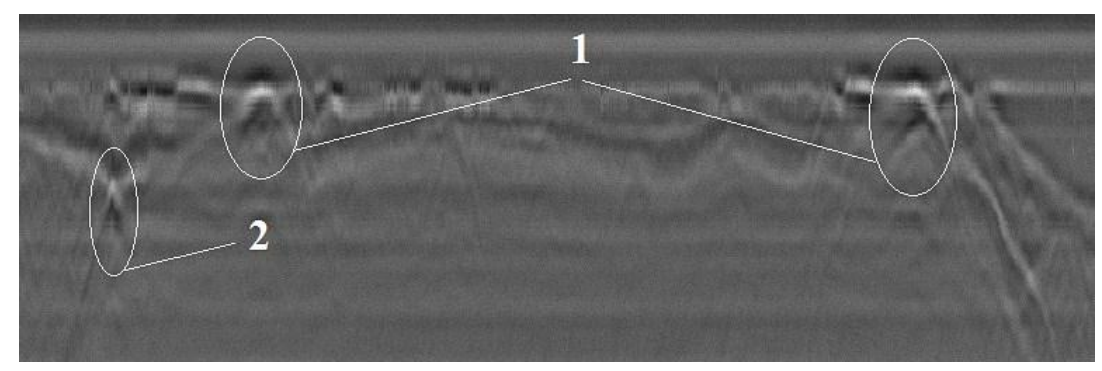

Fig. 2. Radarogram obtained on one of the roads in Ukraine. 


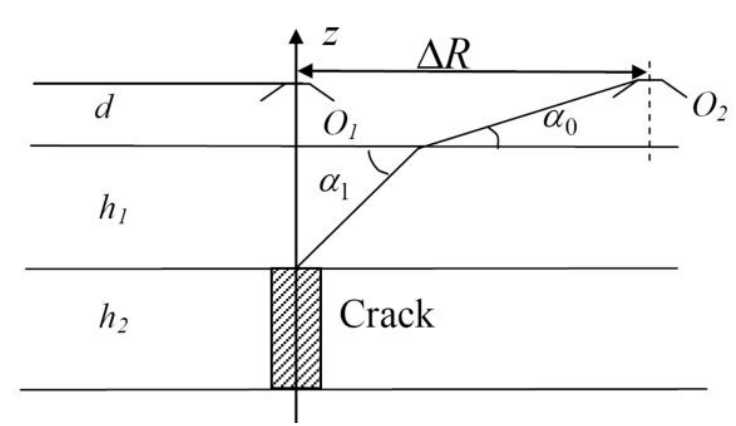

Fig. 3. Geometry of the problem of visual detection of subsurface cracks.

We will assume that the travel time of the signal from the antenna to the upper boundary of the crack in these two cases is known to us and we will designate these quantities $t_{1}$ and, $t_{2}$ respectively. Then, using Snell's law [29], we obtain:

$$
\begin{gathered}
\sin \left(\frac{\pi}{2}-\alpha_{0}\right) \cdot n_{0}=\sin \left(\frac{\pi}{2}-\alpha_{1}\right) \cdot n_{1} ; \\
n_{0}=\sqrt{\varepsilon_{0}}=1 ; \quad n_{1}=\sqrt{\varepsilon_{1}} .
\end{gathered}
$$

Now, in the approximation of geometric optics, we can write down the basic equations for the connection of the known parameters of the problem with the unknown ones:

$$
\left\{\begin{array}{l}
\frac{d}{c}+\frac{h_{1} \cdot \sqrt{\varepsilon_{1}}}{c}=\frac{t_{1}}{2} \\
\frac{d}{\sin \alpha_{0} \cdot c}+\frac{h_{1} \cdot \sqrt{\varepsilon_{1}}}{\sin \alpha_{1} \cdot c}=\frac{t_{2}}{2} \\
\sin \left(\frac{\pi}{2}-\alpha_{0}\right)=\sqrt{\varepsilon_{1}} \cdot \sin \left(\frac{\pi}{2}-\alpha_{1}\right), \\
\left(\varepsilon_{0}=1\right)
\end{array}\right.
$$

where $\mathrm{c}$ is the speed of light in vacuum, $\mathrm{cm} / \mathrm{ns}$. If the dielectric constant of the upper layer is unknown, then the equations of system (2) contain four unknown quantities $\varepsilon_{1}, h_{1}, \alpha_{0}, \alpha_{1}$ and this system, strictly speaking, does not have a unique solution. Various methods have been proposed to further obtain an approximate solution. One of them involves calculating the angle $\alpha_{0}$ value from geometric data and calculating the first term in the second equation of system (2). It is also possible to replace this equation with another approximate equation, which no longer contains the first term on the left:

$$
\frac{h_{1} \cdot \sqrt{\varepsilon_{1}}}{\sin \alpha_{1} \cdot c}=\frac{t_{2}}{2} .
$$

Other approaches to solving this problem involve processing a large amount of information, and some of them are very complex from a computational point of view. Therefore, it becomes necessary to develop more sophisticated signal processing algorithms that would allow not only detecting, but also identifying subsurface cracks.

Therefore, further we will consider the problem of detecting a subsurface crack in the structure of a pavement. Consider the simplest model of the environment, which has three layers located on a semi-infinite substrate (Fig. 1). The use of such a model is due to the fact that the so-called electrical contrast - the ratio of the values of the relative permittivity of the lower layers is weak and the influence of multiple reflections of signals between the boundaries of the lower layers can be neglected. In other words, small differences in the values of the dielectric constant lead to weak amplitudes of signals reflected from the lower boundaries. In addition, the thickness of the lower layers, as a rule, significantly exceeds the thickness of the upper layers, which causes their displacement along the time axis. This makes it possible to ignore reflections from the lower boundaries at the first stage of signal processing. As noted above, the main disadvantage of visual analysis of radarograms is the impossibility of obtaining quantitative estimates of the parameters of cracks. Therefore, earlier the authors proposed an approach to solving the problem of quantitative estimates. This approach includes carrying out measurements and processing the results using specialized programs developed by the authors. The essence of this approach is in the two stages of data processing. At the first stage, standard operations are performed to measure the direct transmission signal, i.e. - powerful direct coupling signal (a signal that directly passed into the receiving antenna, bypassing the probed medium) and search for a homogeneous reference area without any heterogeneity. The algorithm for registering a direct transmission signal is described in [23,25], and the reference section is selected based on the absence of changes in this section of the radarogram. In some cases, this data can be obtained during road construction in order to further use it in the process of searching for defects. After selecting a reference area, the thickness of the pavement layers is estimated. For this, the GeoVizy program is used, which allows in a semi-automatic mode to estimate the values of the dielectric constant and the thickness of the pavement layers. This information is further used to estimate the parameters of the cracks. The problem of detecting and determining the parameters of cracks can be formulated on the basis of various physical principles. In this work, we will consider one of the possible 
implementation options, which is based on the use of only one antenna unit. In other words, the goal of this approach is to use the simplest and most affordable technical means. Of course, this is only possible due to some decrease in the overall quality of the final assessment.

\section{THE PROPOSED PROBLEM-SOLVING METHOD}

The measurements were carried out in the laboratory on a model of the road structure using the Odyag-5 GPR Fig. 4. For convenience, Figure 4 shows only two antennas (transmitting and receiving) and a model of a cracked pavement structure. Several series of measurements were carried out according to the previously proposed scheme, which consisted of measuring:

- direct coupling signal,

- the signal reflected from the sheet of metal and

- signals from the investigated structure.

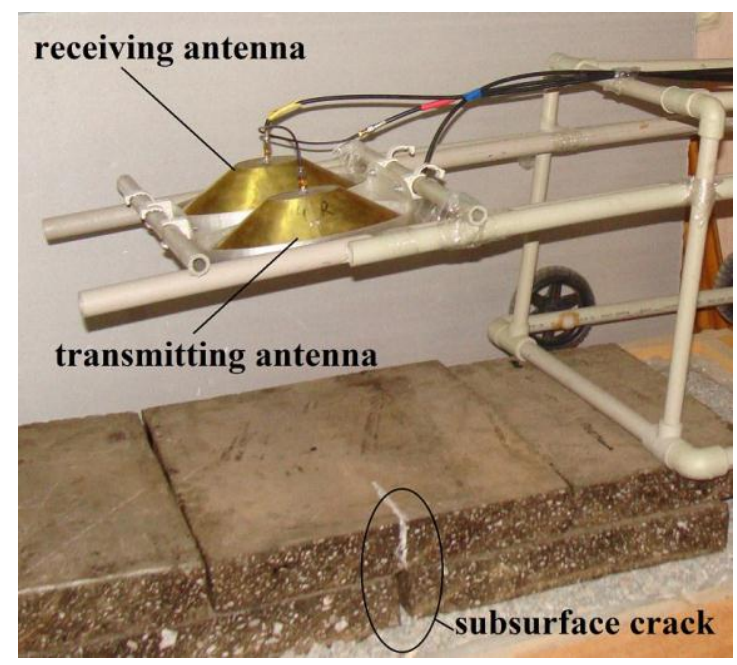

Fig. 4. Experimental setup.

Moreover, as it can be seen from Fig. 4. since the antennas were located in parallel, their vectors of the electric field of the antennas were also parallel. Obviously, during sounding, the crack can be located at different angles to the direction of the vectors of the electric field of the antennas. It is clear that the amplitude of the signal reflected from the crack will depend on the relative orientation of the antennas and the crack. To predict the type of this dependence theoretically is a rather difficult task. This is due both to the complex nature of the interaction of pulsed UWB GPR signals with layered media, and to various geometric configurations and filling of cracks. For example, a crack that is filled with water will reflect the impulse signal much more intensively than a fracture filled with air, due to the difference in dielectric constant between water and air.

It is clear that a road engineer conducting diagnostics must have, first of all, the ability to detect subsurface cracks. Therefore, we first carried out experiments to analyze the detection capabilities of such cracks in laboratory models (Fig. 4.). For verification, a model of a crack $2 \mathrm{~cm}$ wide was used in the lower layer of asphalt concrete with the thickness of $6.5 \mathrm{~cm}$. The upper layer had a thickness of $5.5 \mathrm{~cm}$. (see Fig. 5).

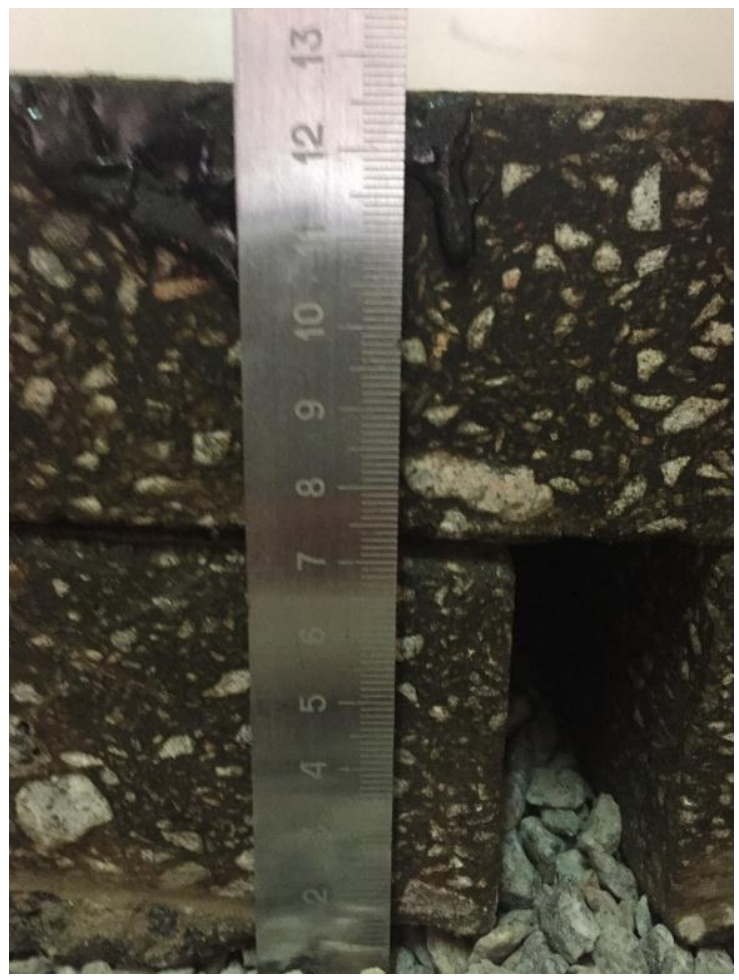

Fig. 5. Dimensions of the investigated structure.

To obtain the initial data, the following scheme was used. After following the standard procedures described above, the vehicle was driven over the structure. Then the antennas were rotated over the crack to analyze the influence of the polarization state of the probing electromagnetic field on the distribution of reflected pulses. The purpose of this was to find the optimal parameters of the probing field for solving the problem of detecting subsurface cracks. The results of the analysis of the polarization state of the probing electromagnetic field can be used not only to improve the signal processing algorithms, but also to create antenna units with switched polarization [25]. We expect that this will be reflected in our subsequent publications.

After registering the data, they were processed using the GeoVizy-2020 software. The view of the program window during data processing is shown in Fig. 6. The basis of the method for processing the pulsed sensing data is the layer-by-layer determination of two parameters - the relative permittivity and the thickness of each layer [23]. This approach is the foundation for the development of the crack detection method proposed in this article. Its essence consists in using the information that was accumulated at the first stages of processing for the subsequent more 
detailed analysis of impulse signals. We emphasize once again that the general idea is to study the possibility of detecting subsurface cracks using the existing standard antenna unit at the expense of some complicating the data registration procedure. This complication consists in carrying out additional measurements while rotating the antenna unit over the crack. It is important that it is not carried out permanently, but only if characteristic differences are found in the radarogram.

To illustrate the proposed algorithm, Fig. 6 shows 3 different signals. They are provided with indices 1 - signal reflected from a sheet of metal, 2 - signal reflected from a structure in an area without a crack, and 4 - a virtual signal created by the GeoVizy-2020 program. Two more signals (3 and 5) are the result of applying the Hilbert transform [30] to signals 2 and 4, respectively.

The GeoVizy-2020 software allows one to process data in a semi-automatic mode. This means that at the first stage, to select the required reference point, it's simply necessary to press the "min" or "max" button, depending on the polarity of the original signal. After that, the program automatically generates virtual signal 4 , which allows to calculate the layer thickness [23]. As a result of data processing at this stage, the program determines the values of two parameters of the upper layer dielectric constant and thickness and displays the results in the windows "h1" and "pmt1" Fig.6. Besides. at the first stage, if necessary, it is possible to subtract the direct transmission signal in a semiautomatic mode. To speed up the processing, GeoVizy-2020 also has the functionality of subtracting the direct transmission signal from the entire profile and then saving the data. At the next stage, the parameters of the second layer were determined. This layer was a layer of asphalt concrete about $6.5 \mathrm{~cm}$ thick and contained a crack model - Fig. 5. To obtain the necessary data, as in the previous stage, a virtual signal synthesis algorithm was used, which made it possible to automatically get the values of the thickness and relative dielectric constant of the next layer. As a result, the calculated values are displayed in the corresponding windows "h2" and "pmt2" Fig7. Then the process can be continued to obtain the data on the next layer. This completes the preparatory stage of the proposed algorithm.

The next step is to conduct a detailed comparative analysis of various signals. To accomplish this task, the GeoVizy-2020 software provides the ability to simultaneously display and analyze several partial signals. With this term we will designate individual countdowns in the coordinates "time - amplitude" (see Fig. 6, 7). The purpose of this analysis is to compare the signals, taking into account the

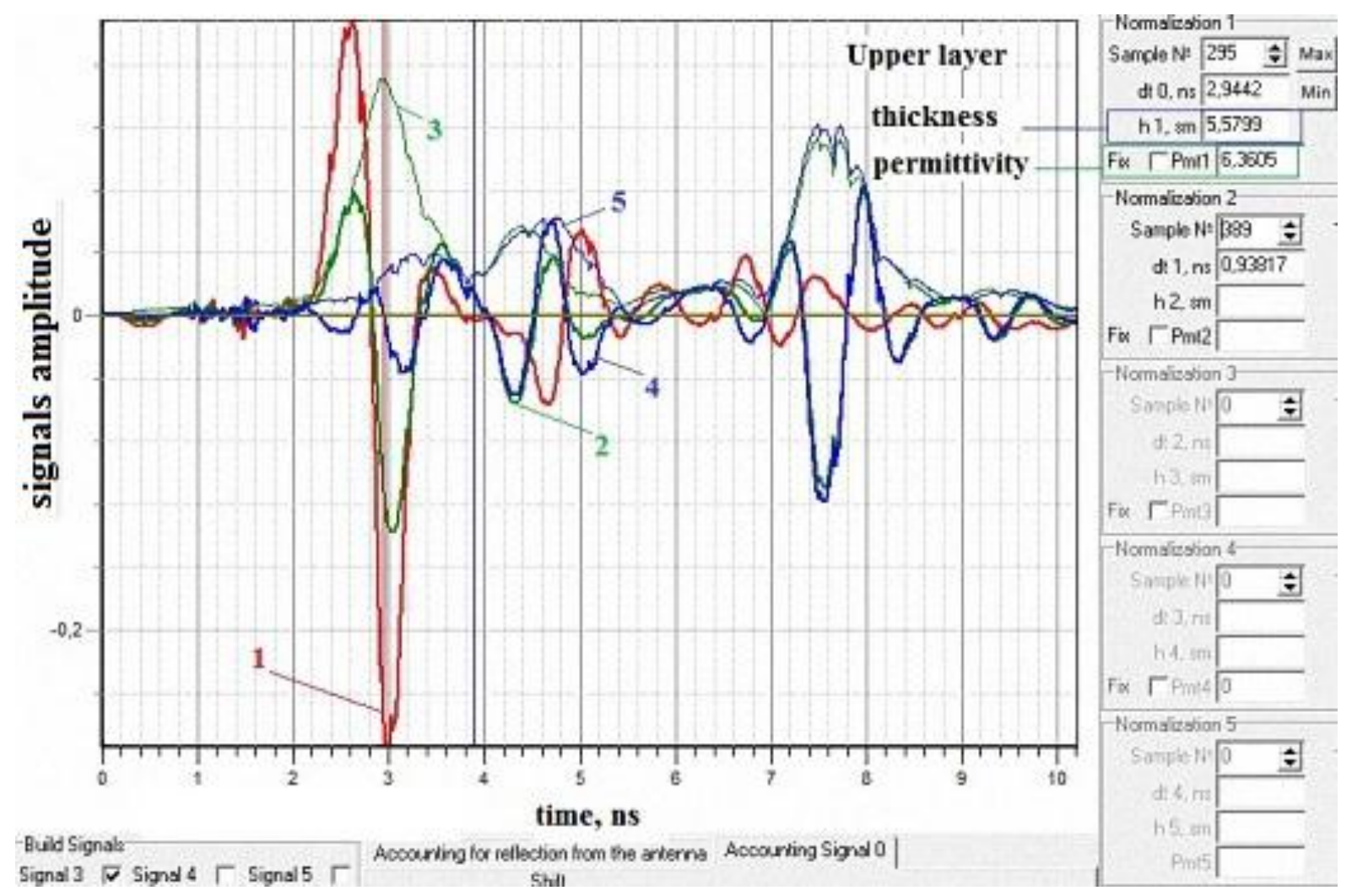

Fig. 6. View of the program window during data processing 


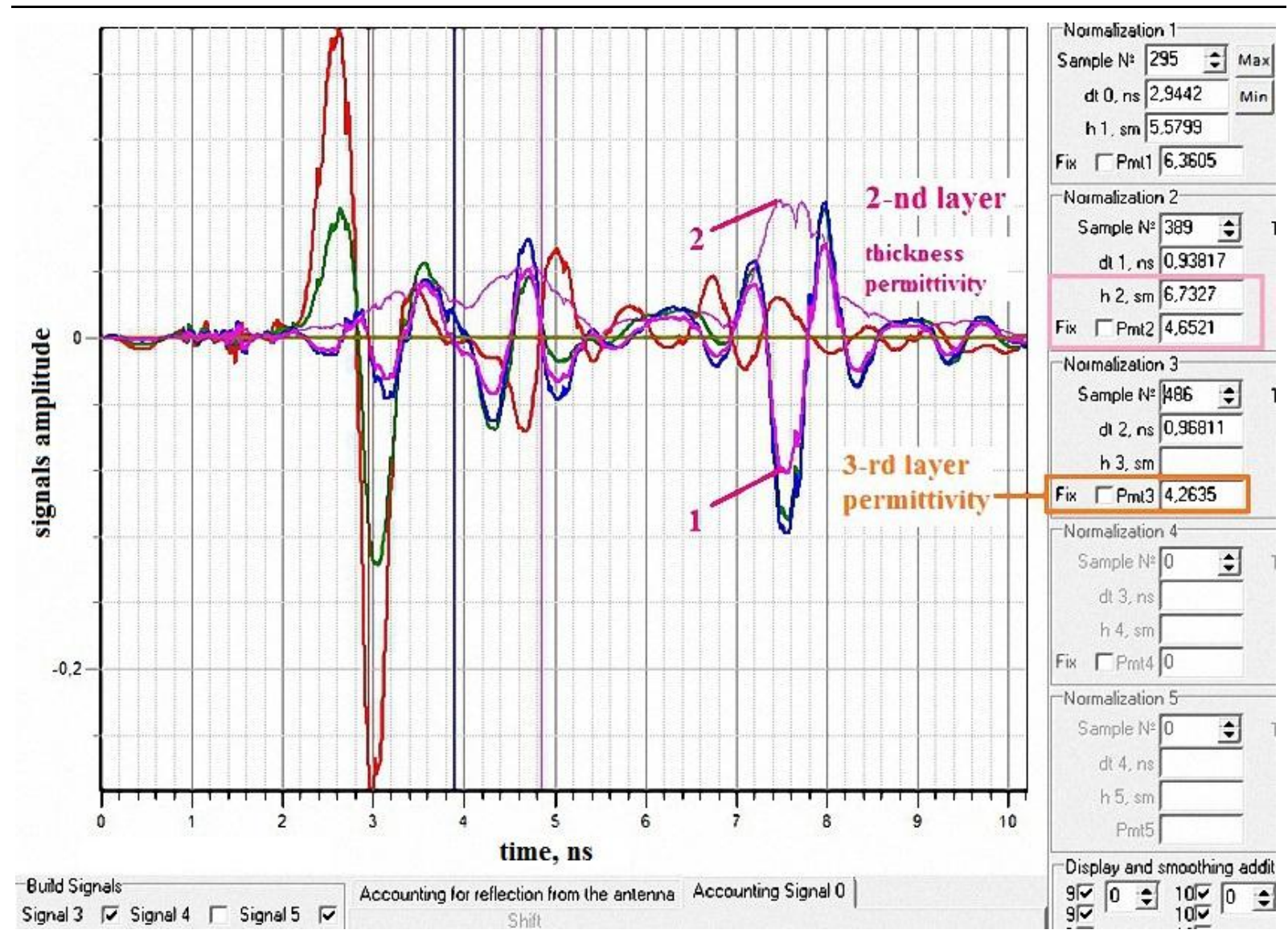

Fig. 7. Data processing window at the next stage

previously obtained information about the thickness of the layers.

The main problem is that subsurface cracks, from the point of view of the electromagnetism theory, have a very weak contrast. Otherwise, due to their small size and relatively small difference in dielectric constant values, they cause a slight change in the reflected signal. Therefore, the main idea of the proposed approach is a targeted search for differences in the shape of signals based on preliminary data on the values of the thickness and dielectric constant of the layers. This algorithm allows the user to focus on certain segments of the distribution of signals in time in order to find the differences. The problem is further complicated by the fact that when the GPR moves along the road surface, small deviations from the initial height of the antenna unit above the surface of the road inevitably occur. This leads to corresponding differences in the waveforms, which are not associated with the presence of subsurface inhomogeneities. Therefore within the framework of this study, laboratory experiments were carried out primarily to analyze the fundamental possibility of detecting low-contrast buried objects. As a result, the possibility of detecting subsurface cracks using the analysis of radarograms was established. It is illustrated in Fig. 8, 9. Fig. 8 shows the graphs of three partial signals without pre-processing. In these figures, the designations of the axes are the same as in the previous ones - Fig. 6.7. These figures clearly illustrate the occurrence of deviations, i.e. signal displacement caused by the movement of the GPR along the surface even in laboratory conditions. The problem is further complicated by the presence of a slight instability of the parameters of the GPR itself. Nevertheless, the results of preliminary processing based on the data on the thickness of the layers made it possible to identify the area of possible presence of subsurface inhomogeneities. Further Fig. 9 shows the results of correcting the initial data by shifting the graphs along the time axis without changing their amplitude.

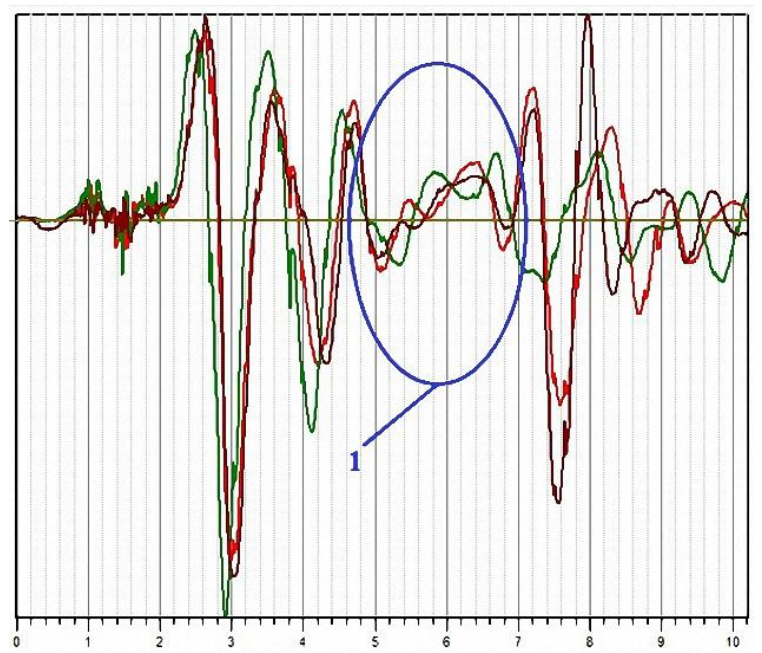

Fig. 8. Three partial signals without pre-processing. 
Analysis of this figure allows us to make a conclusion about the stability of the original signal, caused by reflection from the outer surface of the pavement and small deviations in the amplitude of the signal reflected from the lower surface of the upper layer. This, when measured on real roads, can be associated with a change (variation) in the thickness of the asphalt concrete layers, and when measured in a laboratory, it can be caused by the unevenness of the lower surfaces of the samples and their loose fit in some places.

Finally, in order to investigate additional possibilities of detecting subsurface cracks that a pulsed GPR provides, let us consider some of the features of measurements when the antenna unit rotates over the crack. In the course of these measurements, the GPR antenna unit was turned over the crack so that the distance to the surface remained constant. The results are shown in Table 1. Since the graphs of pulse signals reflected from subsurface inhomogeneities are complex, it was decided to take into account only the change in the maximum signal amplitude. The experiment was carried out in such a way that at the beginning the antennas were positioned as shown in Fig. 4, and then turned to 90 degrees. Table 1 also shows not only the amplitudes

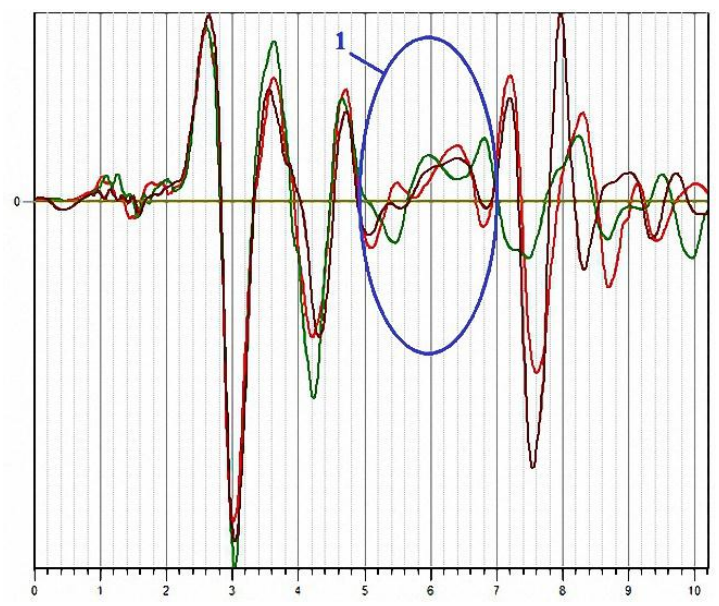

Fig. 9. Results of correcting the initial data.

themselves, but also their deviations from the mean. Analysis of the results in Table 1 taking into account the data in Fig. 8, 9 shows the impossibility of using simple quantitative models to interpret the data obtained. This is due to the dispersion of the dielectric constant (permittivity), as well as to the complex nature of wave processes in the presence of low-contrast subsurface inhomogeneities. In this case, the very fact of the possibility of detecting subsurface inhomogeneities using relatively simple technical means is of prime importance. Of course, the use of more advanced approaches [9, 25] involving the analysis of the polarization state of the probing and reflected signals can further improve the quality of the quantitative estimates obtained.
Table 1. Data obtained

\begin{tabular}{|c|c|c|}
\hline $\begin{array}{c}\text { angle of } \\
\text { rotation }\end{array}$ & $\begin{array}{c}\text { min amplitude in relative } \\
\text { units, taking into account } \\
\text { the sign of the maximum }\end{array}$ & $\begin{array}{c}\text { deviation from the } \\
\text { mean, in relative } \\
\text { units }\end{array}$ \\
\hline 0 & $-1,02$ & 0,56 \\
\hline 20 & $-2,84$ & 0,26 \\
\hline 45 & $-2,08$ & 0,31 \\
\hline 70 & $-2,13$ & 0,23 \\
\hline 90 & $-2,27$ & 0,18 \\
\hline
\end{tabular}

\section{VERIFICATION OF DATA PROCESSING MODELS ADEQUACY.}

As is known from the electromagnetic theory, the relative permittivity of real media has the property of dispersion [31], i.e. it depends on the frequency. Therefore, the procedure for determining the value of the dielectric constant of the layers of the road pavement by measuring the reflection coefficient of pulse signals of ground penetrating radars, generally speaking, requires clarification. At the same time, in an arbitrary case, it is rather difficult to answer the question of accuracy of determining the dielectric permittivity value. Therefore, the most simple and effective method is the proposed method for assessing the thickness of the layers. It is easy to measure this value in the laboratory and then compare the obtained results with the results of processing the GPR data. When conducting surveys on real roads, one can use the results of direct measurements from the cores. The corresponding experiments were carried out and their results are reflected in table 2 . Table 2 reflects the results of the two cases. The first case corresponds to the experiments described above (Fig. 4 - Fig. 7). The second case reflects the results of the measurements on the road in Kharkov region. As one can see, the results of processing the GPR data show a fairly good accuracy of reconstructing the layer thickness. It is clear that due to the accumulation of measurement and processing errors, the accuracy of determining the thickness of the lower layers deteriorates in comparison with the accuracy for the upper layers. However, in general, the results obtained allow us to speak about the advantages of using a GPR for searching for subsurface cracks and assessing the state of the road pavement. A detailed discussion of the results concerning error handling can be found in [23].

Table 2. Results of the two cases.

\begin{tabular}{|c|c|c|}
\hline $\begin{array}{c}\text { measured } \\
\text { thickness value }\end{array}$ & $\begin{array}{c}\text { determined according to } \\
\text { GPR data, in relative } \\
\text { units }\end{array}$ & $\begin{array}{c}\text { percentage } \\
\text { difference }\end{array}$ \\
\hline \multicolumn{3}{|c|}{ Case 1} \\
\hline 5,50 & 5,57 & 1,2 \\
\hline 6,5 & 6,73 & 3,5 \\
\hline \multicolumn{3}{|c|}{ Case 2} \\
\hline 7,2 & 7,01 & 2,7 \\
\hline 8 & 8,24 & 3,0 \\
\hline
\end{tabular}




\section{CONCLUSIONS}

The presented results of laboratory experiments clearly demonstrate capabilities of modern remote sensing technologies. The analysis of the existing remote sensing tools has made it possible to formulate a new approach to solving the urgent problem of detecting hidden subsurface cracks. The proposed approach is based on previously developed algorithms for processing pulsed ground penetrating radar signals $[9,16,24]$. This approach demonstrated the ability to coherently solve several problems. The first task was to select the most optimal technical diagnostic tools with simultaneous laboratory experiments. As a result, the effectiveness of the method was confirmed. Another task was to further improve the methods and the computational algorithms for processing sensing data. As a result of the modernization of the GeoVizy program, the new version of GeoVizy2020 made it possible to process complex impulse signals and realize the ability to display the quantitative values of the most important parameters of the pavement layers - the relative permittivity and thickness. To check the adequacy of the results obtained, we used the method proposed by the authors, based on the procedure for measuring the real thickness of the layers in laboratory and field conditions and comparing these data with the results obtained using numerical simulation. As a result, evidence is presented of the advantages of using impulse georadars for examining road pavements, searching for subsurface cracks and estimating layer parameters.

As prospects for further research in this direction, it should be noted that there are possibilities of improving technical means (antennas, generators), as well as of developing data processing methods regarding not only presence of damage, but also assessment of the current state of the road and forecasts on funding required for repair and maintenance of road network [32].

\section{REFERENCES}

1. Volovski M, Murillo-Hoyos J, Saeed TU, Labi S. Estimation ofroutine maintenance expenditures for highway pavement segments: accounting for heterogeneity using random-effects models. Journalof Transportation Engineering, 2017; Part A: Systems. 143(5) https://doi.org.10.1061/JTEPBS.0000041.

2. Woldemariam W, Murillo-Hoyos J, Labi S. Estimating annual maintenance expenditures for infrastructure: artificial neural networkapproach. Journal of Infrastructure Systems, 2015;22(2):1-9, https://doi.org.10.1061/(ASCE)IS.1943555X.0000280.

3. Adams TM. Estimating Cost per Lane Mile for Routine Highway Operations and Maintenance. National Center for Freight and Infrastructure Research and Education, University of Wisconsin,
Madison, WI, USA, 2011, Technical Report No. MRUTC 07-12.

4. Ground penetrating radar, theory and applications. Elsevier B.V. 2009.

5. Evans RD. Optimising ground penetrating radar (GPR) to assess pavements. A dissertation thesis submitted in partial fulfillment of the requirements for the award of the degree Doctor of Engineering (EngD), at Loughborough University. 2009.

6. Yan Xua, Lijun Sun. Study on permanent deformation of asphalt mixtures by single penetration repeated shear test. Procedia - Social and Behavioral Sciences 96, 13th COTA International Conference of Transportation Professionals (CICTP 2013). https://doi.org/10.1016/j.sbspro.2013.08.101

7. Zulufqar Bin Rashid1, Rakesh Gupta. Study of defects in flexible pavement and its maintenance. International Journal of Recent Engineering Research and Development (IJRERD). 2017; 2(6):30-37. www.ijrerd.com.

8. Mezgeen AR, Vega Pérez-Gracia, Francisco MF, Jorge CP, Sonia Santos-Assunçaoa Caio Santos, Viviana Sossa. GPR laboratory tests and numerical models to characterize cracks in cement concrete specimens, exemplifying damage in rigid pavement. Measurement. 2020;158:107662

https://doi.org/10.1016/j.measurement.2020.107662.

9. Batrakov DO, Antyufeyeva MS, Antyufeyev AV, Batrakova AG. GPR data processing for evaluation of the subsurface cracks in road pavements // 2017 9th International Workshop on Advanced Ground Penetrating Radar (IWAGPR). 2017. http://ieeexplore.ieee.org/document/7996072/

10. Carl Lenngren, Ebrahim Parhamifar. FWD Testing as a Construction Control Tool. 9th International Conference on the Bearing Capacity of Roads, Railroads, and Airfields. 2013; 1:1-11.

11. Payntar G. Method of test for obtaining deflection measurements and layer thickness information for rehabilitation design of hot mix asphalt surfaced pavements using mechanistic-empirical design and analysis procedures. State of California-business, transportation and housing agency, department of transportation division of engineering services Transportation Laboratory 5900 Folsom Boulevard Sacramento, California. 2014; 112. https://dot.ca.gov/-/media/dot-media/programs lengineering/documents/californiatestmethodsctm/ctm-357-a11y.pdf

12. Gudmarsson A, Ryden N, Di Benedetto H, Sauzéat C, Tapsoba N, Birgisson B. Comparing linear viscoelastic properties of asphalt concrete measured by laboratory seismic and tension-compression tests. Journal Nondestruct Eval. 2014;33:571-582. https://doi.org/10.1007/s10921-014-0253-9.

13. Nils Ryden. Seismic pavement testing. International Conference on Engineering Geophysics. At: Al Ain, United Arab Emirates. 2015: 1-5. https://doi.org/10.1190/iceg2015-005.

14. Park C, Ivanov J, Miller RD, Xia J. Seismic Investigation of Pavements by MASW Method Geophone Approach. Geophysics to Engineering and Environmental Problems. 2001:1-9. https://doi.org/10.4133/1.2922938.

15. Henrik Bjurström. Non-contact surface wave measurements on pavements. Doctoral Thesis, KTH Royal Institute of Technology, School of Architecture and the Built Environment, Department 
of Civil and Architectural Engineering, Division of Soil and Rock Mechanics, SE-114 28 Stockholm. 2017. https://www.diva-portal.org/smash/get/diva2: 1073021/FULLTEXT01.pdf

16. Batrakov DO, Batrakova AG, Antyufeyeva MS. Combined GPR data analysis technique for diagnostics of structures with thin near-surface layers. Diagnostyka. 2018; 19(3):11-20. https://doi.org/10.29354/diag/91489.

17. Zehua Dong et al. Rapid detection methods for asphalt pavement thicknesses and defects by a vehicle-mounted ground penetrating radar (GPR) system. Sensors (Basel). 2016;16(12):2067. https://doi.org/10.3390/s16122067

18. Batrakov DO. Antyufeyeva MS, Antyufeyev OV, Batrakova AG. GPR application for the road pavements surveys./ 2017 IEEE Microwaves, Radar and Remote Sensing Symposium (MRRS). 2017: 8184.

19. Ground penetrating radar, theory and applications. Amsterdam. Elsevier B.V. 2009.

20. Evans RD. Optimising ground penetrating radar (GPR) to assess pavements. A dissertation thesis submitted in partial fulfillment of the requirements for the award of the degree Doctor of Engineering (EngD), at Loughborough University. 2009.

21. Batrakov DO, Antyufeyeva MS, Antyufeyev AV, Batrakova AG. Remote sensing of plane-layered media with losses using UWB signals. XI International Conference on Antenna Theory and Techniques (ICATT), 2017: 370-373. IEEE Conference Publications. http://ieeexplore.ieee.org/document/7972666/

22. Lachowicz J, Rucka M. Numerical modeling of GPR field in damage detection of a reinforced concrete footbridge.Diagnostyka. 2016;17(2):3-8.

23. Pochanin, GP, Masalov SA, Ruban VP, Kholod PV, Batrakov DO, Batrakova AG, Urdzik SN, Pochanin OG. Advances in short range distance and permittivity ground penetrating radar measurements for road surface surveying, in Advanced Ultrawideband Radar: Signals, Targets and Applications. CRC Press - Taylor \& Francis Group. 2016.

24. Batrakov DO, Antyufeyeva MS, Batrakova AG, Antyufeyev AV. UWB signal processing for the solving inverse scattering problem of plane-layered media. Proceedings of the 9th International Conference on Ultrawideband and Ultrashort Impulse Signals (UWBUSIS-2018). 2018:140-143. https://ieeexplore.ieee.org/document/8520255/

25. Batrakov DO, Batrakova AG, Pochanin GP, Orlenko OA. Method of detection and determination of direction, including subsurface cracks in asphalt concrete pavements. Patent for the invention №118409, Ukraine, 10. 01. 2019.

26. Mezgeen A. Rasol Vega Pérez-Gracia, Mercedes Solla, Jorge C. Pais, Francisco M. Fernandes, Caio Santos, Sam Roberts. Early cracking observation in road pavements with Ground Penetrating Radar: Field and numerical study. N Conference: 1st International Conference On Ground Penetrating Radar Applications For Solving Engineering Problems. At: Wroclow. 2019.

27. Pochanin GP, Ruban VP, Batrakova AG, Urdzik SN, Batrakov DO. Measuring of thickness of asphalt pavement with use of GPR. 15th International radar symposium . proceedings (Gdansk, Poland, June 16-
18, 2014). - Warsaw University of Technology. 2014:452 -455.

28. Tian Xia, Dryver Huston. High speed ground penetrating radar for road pavement and bridge structural inspection and maintenance. Final Report. Project Number: SPR-RSCH017-738. Report Submitted on: 06/30/2016.

https://vtrans.vermont.gov/sites/aot/files/highway/doc uments/materialsandresearch/completedprojects/Final \%20Report\%20-\%20738\%20\%20High\%20Speed\%20GPR\%2010-17-2016.pdf

29. Born M, Wolf E. Principles of Optics. Electromagnetic theory of propagation, interference, and diffraction of light. Pergamon, London; Macmillan, New York, ed. 2. 1964.

30. David Ernesto Troncoso Romero, Gordana Jovanovic Dolecek. Digital FIR hilbert transformers: fundamentals and efficient design methods. A Fundamental Tool for Scientific Computing and Engineering Applications - Volume 1. InTech-Open. 2012. https://doi.org/10.5772/46451.

31. Pasquini B, Vanderhaeghen M. Dispersion theory in electromagnetic interactions. Annual Review of Nuclear and Particle Science. 2018;68:75-103. https://doi.org/10.1146/annurev-nucl-101917020843.

32. Batrakova AG, Troyanovsky VV, Batrakov DO, Pilicheva MO, Skrypnyk NS. Prediction of the road pavement condition index using stochastic models. Roads and Bridges - Drogi i Mosty. 2020;19(3): 225242. https://doi.org/10.7409/rabdim.020.015.

\section{Received 2020-09-11}

Accepted 2021-05-20

Available online 2021-05-21

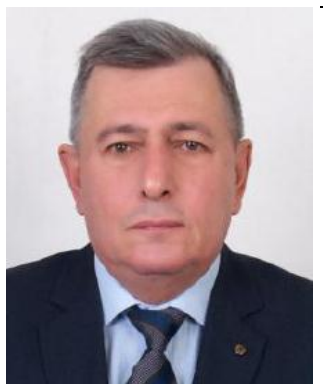

Dmitry O. BATRAKOV- Dr. Sci., Full Professor - Radiophysics, biomedical electronics and computer systems faculty, Chair of Theoretical Radiophysics, V. Karazin National University, Kharkiv, Ukraine.

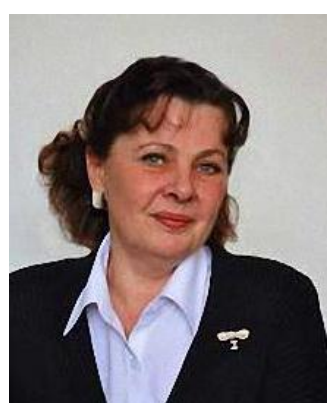

Dr Angelika G. Batrakova Dr. Sci., Full Professor - Chief of the Chair of Highway Design, Geodesy and Land Management, Kharkiv National Automo-bile and Highway University, Kharkiv, Ukraine.

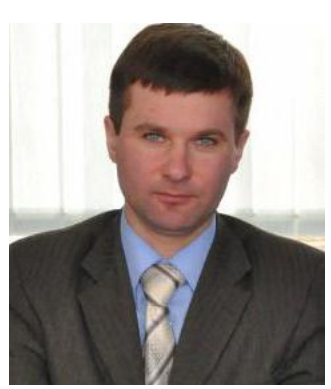

Sergey N. Urdzik, assistant of professor, Chair of Highway Design, Geodesy and Land Management, Kharkiv National Automobile and Highway University, Kharkiv, Ukraine. 
Batrakov DO, Batrakova AG, Urdzik SN, Danielyan VR.: Nondestructive diagnostics and detection of ...

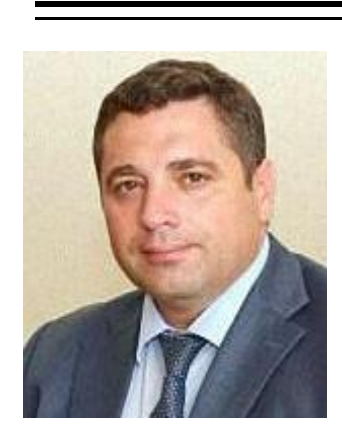

Vadim R. Danielyan postgraduate student, Chair of

Highway Design, Geodesy and

Land Management, Kharkiv

National Automobile and

Highway University, Kharkiv,

Ukraine. 